\title{
Association of Severe Noncerebral Plasmodium falciparum Malaria in Brazil With Expressed PfEMPI DBL1 $\alpha$ Sequences Lacking Cysteine Residues
}

\author{
Karin Kirchgatter ${ }^{1,2}$ and Hernando A. del Portillo ${ }^{1}$ \\ ${ }^{1}$ Departamento de Parasitologia, Instituto de Ciências Biomédicas, Universidade de São Paulo, \\ São Paulo, Brazil \\ ${ }^{2}$ Núcleo de Estudos em Malária, Superintendência de Controle de Endemias (SUCEN), São Paulo, \\ São Paulo, Brazil
}

Accepted December 13, 2001

\begin{abstract}
Background: Cytoadherence and rosetting contribute to the development of severe Plasmodium falciparum malaria. In Brazil, severe falciparum malaria is mostly associated with renal or pulmonary complications and very rarely with cerebral malaria. The most $\mathrm{N}$-terminal DBLl $\alpha$ domain of PfEMP1, a protein encoded by the var multigene family mediates rosetting. We analyzed parasites of Brazilian patients with severe malaria to determine whether there were particular DBLl $\alpha$ var sequences predominantly expressed in such patients.

Materials and Methods: DBLl $\alpha$ var sequences were obtained from parasites of Brazilian patients with severe and mild malaria and were analyzed by standard bioinformatic programs. Three hundred twenty var DBLl $\alpha$ sequences obtained from 80 Brazilian patients with mild malaria were spotted in high-density filters and hybridized to probes representing predominantly expressed
\end{abstract}

sequences in parasites from patients with severe malaria. A DBL $1 \alpha$ domain was expressed in bacteria and used to demonstrate its binding capacity to erythrocytes by immunofluorescence.

Results: Forty-three different and unreported DBLl $\alpha$ amino acid sequences were obtained. Sequences predominantly expressed in patients with severe malaria could be subgrouped due to deletions of 1-2-cysteine residues. These sequences were commonly found in the var gene repertoire of parasites from patients with mild malaria, yet they were rarely expressed in these patients. A recombinant protein representing the most abundantly expressed sequence detected in one patient with severe malaria bound directly to uninfected erythrocytes.

Conclusion: This is the first report showing an association of severe noncerebral malaria from Brazil with particular DBL $1 \alpha$ sequences.

\section{Introduction}

Plasmodium falciparum is the most virulent and devastating human malarial parasite and is responsible for approximately 300 million annual clinical cases and 1 million deaths, mostly in children under 5 years old. Falciparum infections are characterized by removal from the peripheral circulation of red blood cells infected with mature parasites. This sequestration occurs by adhesion of infected erythrocytes to host receptors in the microvasculature of several organs. This phenotype, termed cytoadherence, cause retention and accumulation of infected erythrocytes leading to occlusion of microvessels and acute pathologic changes associated with severe malaria (1). Cytoadherence is conferred by the $P$. falciparum erythrocyte membrane protein 1 (PfEMP1), encoded by a multigene family termed var, which has a two exon

Address correspondence and reprint requests to: H.A. del Portillo, Departamento de Parasitologia, Instituto de Ciências Biomédicas, Universidade de São Paulo, Av. Lineu Prestes 1374, São Paulo-SP, 05508-900 Brazil. Phone: +55-11-3091-7209; fax: +55-11-3091-7417; e-mail: hernando@icb.usp.br structure: a conserved acidic cytoplasmic tail (ATS) and a highly polymorphic $\mathrm{N}$-terminus displaying variant numbers of Duffy binding-like (DBL) domains (2). It is within the highly polymorphic $\mathrm{N}$ terminus that several ligands for different endothelial receptors have been mapped (reviewed in 3 ).

In addition to cytoadherence, it is now widely accepted that the adhesion of infected erythrocytes to uninfected erythrocytes, a phenomenon called rosetting, further contributes to the pathology associated with falciparum infections (4). Indeed, rosetting has been associated with severe malaria; it is common in parasites from patients with malaria complications in many field studies (5-11). Moreover, several lines of evidence have demonstrated that the most N-terminal DBLl $\alpha$ of PfEMPI mediates rosetting (12-15). Thus, PfEMPl plays a major role in cytoadherence and rosetting. Both phenomena are directly associated with severe falciparum malaria.

Severe falciparum malaria is a complex of different clinical manifestations leading mainly to cerebral, renal, and pulmonary dysfunction. In many parts of 
the world, cerebral dysfunction is the more common severe manifestation causing death in $4-50 \%$ of the adults with severe malaria (16). In regions such as Brazil, however, severe falciparum malaria is mostly associated with renal and pulmonary complications and very rarely with cerebral malaria (17-19). Interestingly, a recent report has associated severe malaria in patients from French Guyana and particular var DBL $\delta$ sequences, indicating that isolates from individuals with different clinical syndromes could be characterized by particular PfEMPI repertoires (20). Only one study is presently available on DBLl $\alpha$ genomic sequences from Brazilian isolates (21). Here, we analyzed expressed var DBLl $\alpha$ sequences of parasites obtained from patients with severe malaria in the Brazilian Amazon to determine whether there were particular DBLl $\alpha$ sequences predominantly expressed in such patients.

\section{Materials and Methods}

Patients With Severe Malaria and P. falciparum Samples

We investigated six patients diagnosed and treated between 1987 and 2000 in different hospitals in the capital city of São Paulo, Brazil (Table 1). All patients were classified with severe falciparum malaria according to WHO parameters (16). Procalcitonin (PCT) serum levels, recently implicated as a new parameter to diagnose malaria severity (22), were determined using an immunochromatographic test (Brahms PCTQ kit). After obtaining informed consent, blood samples were obtained and stored in liquid nitrogen. All patients had parasitemias $>2 \%$ of $P$. falciparum ring forms in the peripheral circulation and half presented peripheral schizonts further supporting the severity of their infections. Other laboratory data from these patients are reported in Table 2.

\section{Patients With Mild Malaria and P. falciparum Samples}

Eighty patients (20 from each different region as described below) with mild malaria were also included in this study. Samples were collected in different years and from different regions of the Brazilian Amazon: Mato Grosso (1997), Pará (1987-1997), Rondônia (1997), and Acre (1999). In addition, isolates NGl to NG4 obtained from four patients were used to determine expressed var DBLl $\alpha$ sequences (see Table 1). Blood samples were collected and stored as described above.

\section{In Vitro Culture and Purification of Mature Parasites}

Parasites were cultured in vitro (23) and parasite growth monitored by Giemsa staining of thin and thick blood films. Parasites obtained from blood of patients with severe malaria were matured for one cycle to trophozoites and schizonts, and those from patients with mild malaria, with lower parasitemias (see Table 1), were maintained in culture for up to three cycles. Microscopic analysis of Giemsa-stained smears from all samples confirmed that they all had $<1-3 \%$ of ring stages. Mature parasites obtained from all patients were purified as described elsewhere (24).

\section{DNA/RNA Extractions and cDNA Synthesis}

Genomic DNA was obtained as described in Ferreira et al (25) and stored at $4^{\circ} \mathrm{C}$. Total RNA from mature parasites was extracted according to Kyes et al (26) and stored at $-70^{\circ} \mathrm{C}$. As needed, $1 \mu \mathrm{g}$ aliquots of total RNA were treated in three consecutive 15-min

Table 1. Isolates from severe and mild falciparum malaria, epidemiologic data, parasitemia, and PCT concentrations

\begin{tabular}{|c|c|c|c|c|c|}
\hline Isolate & $\begin{array}{l}\text { Brazilian State } \\
\text { of Origin }\end{array}$ & $\begin{array}{c}\text { Previous Malaria } \\
\text { Attacks }\end{array}$ & $\begin{array}{c}\text { Days Between Symptoms } \\
\text { and Treatment }\end{array}$ & Parasitemia & $\begin{array}{l}\text { Serum Concentration } \\
\text { Range of PCT (ng/ml) }\end{array}$ \\
\hline \multicolumn{6}{|l|}{ Severe } \\
\hline G33 & Rondônia & 0 & 5 & $>2.0 \%$ ** & $\geq 2.0$ \\
\hline G23 & Rondônia & 0 & 5 & $>2.0 \%$ & $\geq 0.5$ \\
\hline G34 & Pará & 1 & 10 & $>2.0 \% * *$ & Not determined \\
\hline $\mathrm{G} 2$ & Mato Grosso & 0 & 14 & $>2.0 \%$ & $\geq 2.0$ \\
\hline G29 & Rondônia & 0 & 9 & $>2.0 \%$ ** & Not determined \\
\hline G3 & Mato Grosso & 3 & 3 & $>2.0 \%$ & $\geq 2.0$ \\
\hline \multicolumn{6}{|l|}{ Mild } \\
\hline NGl & Rondônia & 1 & 4 & $0.9 \%$ & $<0.5$ \\
\hline NG2* & Mato Grosso & 4 & Not determined & $0.5 \%$ & $<0.5$ \\
\hline NG3 & Mato Grosso & 4 & Not determined & $0.4 \%$ & $<0.5$ \\
\hline NG4 & Mato Grosso & 2 & 6 & $1.0 \%$ & $\geq 2.0$ \\
\hline
\end{tabular}

*Recrudescence of G3.

**With peripheral schizontaemia.

PCT, procalcitonin. 
Table 2. Laboratory data of the severe malaria patients upon hospital admission

\begin{tabular}{|c|c|c|c|c|c|c|}
\hline Isolate/Age (yrs)/Sex & G33/20/ ð̊ & G23/18/ $q$ & G34/45/ ొ̂ & G2/37/ $\% *$ & G29/41/ ठै & G3/18/ ð̊ \\
\hline Admitted-discharged (dd/mm/yy) & $03-10 / 11 / 87$ & $22-29 / 06 / 87$ & $17-25 / 08 / 89$ & $13 / 01 / 93-03 / 02 / 93$ & $20-28 / 09 / 88$ & $20-27 / 02 / 92$ \\
\hline $\mathrm{pH}(7,35-7,45)$ & 7.361 & & & & 7.343 & 7.360 \\
\hline $\mathrm{PO}_{2}(80 \mathrm{~mm} \mathrm{Hg})$ & 71.5 & & & & $89.5^{\#}$ & 74.6 \\
\hline $\mathrm{PCO}_{2}(40 \mathrm{~mm} \mathrm{Hg})$ & 25.9 & & & & 24.6 & 34.8 \\
\hline $\mathrm{HCO}_{3}(24 \mathrm{mEq} / 1)$ & 15.0 & & & & 13.4 & 19.5 \\
\hline Base excess $(-2$ to $+2 \mathrm{mEq} / \mathrm{l})$ & -8.4 & & & & -10.0 & -4.7 \\
\hline Sat $\mathrm{O}_{2}(>96 \%)$ & & & & & 96.0 & 94.5 \\
\hline Creatinine $(0.4-1.4 \mathrm{mg} / \mathrm{dl})$ & 1.0 & 0.7 & 0.8 & 11.1 & 0.9 & 0.9 \\
\hline Urea $(10-50 \mathrm{mg} / \mathrm{dl})$ & 42 & 24 & 23 & 234 & 60 & 56 \\
\hline Leukocytes $\left(3.5-12,000 / \mathrm{mm}^{3}\right)$ & 6,300 & 9,600 & 6,900 & 6,600 & 5,200 & 6,600 \\
\hline Erythrocytes $\left(4.5-5,500,000 / \mathrm{mm}^{3}\right)$ & & $2,800,000$ & $3,800,000$ & $3,100,000$ & $2,800,000$ & \\
\hline Hemoglobin ô $(>14 \mathrm{~g} / \mathrm{dl})$; $q$ (>12g/dl) & 12.8 & 8.8 & 11.2 & 8.1 & 8.7 & 10.3 \\
\hline Hematocrit $(40-54 \%)$ & 41 & 25 & 35 & 25 & 25 & 33 \\
\hline Platelets $\left(100-500,000 / \mathrm{mm}^{3}\right)$ & 22,500 & 56,000 & & & 64,000 & 29,000 \\
\hline Total serum bilirubin $(<1.5 \mathrm{mg} / \mathrm{dl})$ & & & 2.7 & 3.6 & & 2.8 \\
\hline Direct serum bilirubin $(<0.5 \mathrm{mg} / \mathrm{dl})$ & & & 0.4 & 2.6 & & 1.1 \\
\hline
\end{tabular}

*Patient under peritoneal dialysis procedure.

\#With $\mathrm{O}_{2}$.

steps at room temperature with DNase I (Life Technologies, Carlsbad, CA, USA) reaching a final volume of $20 \mu \mathrm{l}$. The reaction was stopped by adding EDTA to a final concentration of $2.5 \mathrm{mM}$ and incubation at $65^{\circ} \mathrm{C}$ for $10 \mathrm{~min}$. One-microliter aliquots were used in PCR reactions using var DBLl $\alpha$ oligonucleotides (27); only samples that did not amplify were subsequently used in reverse transcription. cDNAs were prepared using the First-Strand cDNA Synthesis Kit (Amersham Biosciences, Little Chalfont, Buckinghamshire, England), and random hexadeoxynucleotides. Samples without reverse transcriptase were used in all reactions as yet another control of DNA contamination.

\section{Polymerase Chain Reaction, Cloning, and Sequencing}

Samples were amplified by polymerase chain reaction (PCR) as previously described (27). PCR or RT-PCR fragments were resolved in $1 \%$ agarose gel, purified (28), and cloned into the pGEM-T Easy Vector (Promega, Madison, WI, USA). Sequence was contracted and obtained from GATC GmbH (Konstanzs, Germany).

\section{Sequence Analysis}

Sequences were translated to amino acids by EditSeq Programme (DNAstar package) and submitted to BLASTP version 2.1.3 (29). Alignments were done using ClustalX version 1.81 (30). Maximum likelihood tree was constructed utilizing the GTR $+\mathrm{I}+\mathrm{G}$ model, selected by Modeltest Program version 3.06 (31). The value of the gamma distribution with shape parameter $\alpha$ was 1.6315 . The quartet puzzling method was used to choose the better likelihood tree (32). All analyses were undertaken with PAUP* 4.06 (33).

\section{Expression of a var DBL1 $\alpha$ Domain as a His-Tagged} Bacterial Protein

The var gene segment coding for the DBLl $\alpha$ Nterminal domain from the $P$. falciparum isolate G2 (clone 1) was subcloned into the pRSET-C Vector (Invitrogen, Carlsbad, CA, USA). Authenticity of the recombinant plasmid was confirmed by DNA sequencing. After IPTG induction, the protein was expressed in Escherichia coli strain BL2 1 at $37^{\circ} \mathrm{C}$ for $2 \mathrm{hr}$ and purified using the basic protocol of the HisTrap Kit using $8 \mathrm{M}$ of urea and according to the manufacturer's instructions (Amersham Biosciences).

\section{Immunofluorescence}

After washing three times in PBS, thin blood smears were prepared with $0.5 \%$ blood group $\mathrm{A} \mathrm{Rh}^{+}$erythrocytes. Erythrocytes were fixed with acetone and 15- $\mu$ l aliquots of two His-tagged bacterial proteins, DBLl $\alpha$-His and Metal-His (an antigen from Leishmania used as a negative control) both at $80 \mu \mathrm{g} / \mathrm{ml}$ in PBS were dropped separately onto the smears and incubated for $30 \mathrm{~min}$ at room temperature. As a positive control, $15 \mu \mathrm{l}$ of an anti-human erythrocyte polyclonal mouse antibody were used. Slides were washed three times with PBS and binding was detected using an IgG2 monoclonal antibody against the His tag (Amersham Biosciences) and ImmunoPure FITC conjugate goat anti-mouse IgG (Pierce, Rockford, IL, USA). 


\section{High-density Filters and Southern Hybridizations}

Plasmid DNA from 320 individual clones was spotted in duplicates onto $12 \times 8 \mathrm{~cm}$ nylon Hybond $\mathrm{N}^{+}$ membranes (Amersham) by a robotic system (Flexys, Genomic Solutions, Ann Harbor, MI, USA) using a 96-pin head. Gridding density was two identical sets of $(4 \times 4) \times 96$. RT-PCR fragments were labeled using random primer with $\left[\alpha^{33} \mathrm{P}\right]$ CTP using the Multiprime DNA Labelling System (Amersham Biosciences) and T7 oligonucleotide was labeled with $\left[\gamma^{33} \mathrm{P}\right]$ ATP using T4 polynucleotide kinase. Membranes hybridized with $\mathrm{T} 7$ were washed in 6XSSC $/ 0.5 \%$ SDS at $37^{\circ} \mathrm{C}$ for $15 \mathrm{~min}$ followed by another wash in the same solution at $5^{\circ} \mathrm{C}$ below Tm for $10 \mathrm{~min}$. Membranes hybridized with var probes were washed at $2 \times \mathrm{SSC}$ for $10 \mathrm{~min}$ at room temperature followed by two washes at $0.1 \times \mathrm{SSC} / 0.1 \%$ SDS at $65^{\circ} \mathrm{C}$ for 15 min each.

\section{Results}

P. falciparum DBL1 $\alpha$ var Sequences Expressed in Patients With Severe Malaria Revealed Predominantly Expressed Sequences Lacking 1-2 Cysteine Residues

Fifty-seven DBLl $\alpha$ var RT-PCR fragments amplified from total RNA of mature parasites obtained from six patients with severe malaria were cloned and sequenced. A total of 25 different deduced amino acid sequences were obtained and compared in blast analysis to available sequences at GenBank. Two sequences had $100 \%$ identity with sequences already deposited: G29-7 with a var gene from a Vanuatu isolate (AF221773) and G3-R1 with a var gene from FCR3 strain (AJ133811). The other 23 sequences were unreported (GenBank accession numbers AF368922 to AF368944). Alignment of all 25 sequences showed that 8 sequences could be subgrouped due to deletions of 1-2 cysteine residues at their C-termini (Fig. 1). These 8 sequences represent 3 sequences from patient G2 and one sequence from each severe patient (G3, G23, G29, G33, and G34). Significantly, these sequences were the most abundantly expressed in each patient as reflected by the large percentage $(>33 \%)$ of identical clones sequences from each sample. These data demonstrate that there are P. falciparum DBLl $\alpha$ sequences lacking 1-2 cysteine residues that are predominantly expressed in parasites obtained from all patients with severe malaria included in this study.

DBL $1 \alpha$ var Sequences Predominantly Expressed in Parasites Obtained from Severe Malaria Patients are Rarely Expressed in Parasites from Mild Malaria Patients

Having identified DBLl $\alpha$ var sequences lacking 1-2 cysteine residues predominantly expressed in all patients with severe malaria from the Brazilian Amazon, we next examined DBLl $\alpha$ var sequences expressed in parasites obtained from patients with mild malaria. Fifty-four DBLl $\alpha$ var sequences were obtained from parasites of four patients with mild malaria (see Table 1). Twenty-one different amino acid sequences were obtained from these patients. Sequence NG4-3 had 100\% identity with varH sequence obtained from the Brazilian ItG2 isolate (AF275864). The other 20 sequences were unreported and submitted to GenBank (accession numbers AF368945 to AF368950 and AF416572 to AF416585). It is important to recall that we included the same patient at the moment of having severe malaria (G3) and 20 days later at the moment of a recrudescence diagnosed as mild malaria (NG2).

Phylogenetic Analysis of DBL $1 \alpha$ Sequences

Phylogenetic analysis of the DBLl $\alpha$ domain from 46 different expressed var genes from Brazil ( 25 from severe and 21 from mild malaria) and 21 sequences from this same domain from Sudan obtained from GenBank was performed. Remarkably, the same sequences that had been subgrouped in the analysis of DBLl $\alpha$ sequences from patients with severe malaria remained subgrouped. Two sequences expressed in parasites from patients with mild malaria and one sequence from Sudan also subgrouped in this branch of the tree (see Fig. 1).

\section{DBL1 $\alpha$ var Sequences Expressed in Parasites Obtained from Patients With Severe Malaria are Frequently Present in the var Repertoire of Parasites Obtained from Patients With Mild Malaria}

We then investigated whether the most predominantly expressed DBLl $\alpha$ var sequences from patients with severe malaria were present in the var gene repertoire of patients with mild malaria in the Brazilian Amazon. To address this, high-density filters were spotted with 320 clones representing DBLl $\alpha$ var sequences of parasites obtained from 80 patients with mild malaria and screened with different probes. First, we used a plasmid-specific probe (T7) to verify whether all the samples were spotted in approximately the same quantity (Fig. 2A). Second, we used single probes representing the most frequently expressed sequences from three individual patients and demonstrated that they are present in the genomic repertoire of many isolates from patients with mild malaria (Fig. 2B, 2C, and 2D). Sequence analysis of several randomly chosen positive clones confirmed that they were identical to the sequence of the probe with which they reacted (data not shown). Similar results were obtained when filters were hybridized with complex RT-PCR probes from individual patients with severe malaria (data not shown). These data demonstrate that the sequences preferentially expressed in parasites from patients with severe malaria are amply present in the genomes of parasites from patients with mild malaria; however, they are rarely expressed in the latter.

\section{A DBL1 $\alpha$ Domain Expressed in Bacteria Binds Directly to Erythrocytes}

Rosetting has been associated with severe malaria and is unequivocally mediated by particular sequences of 

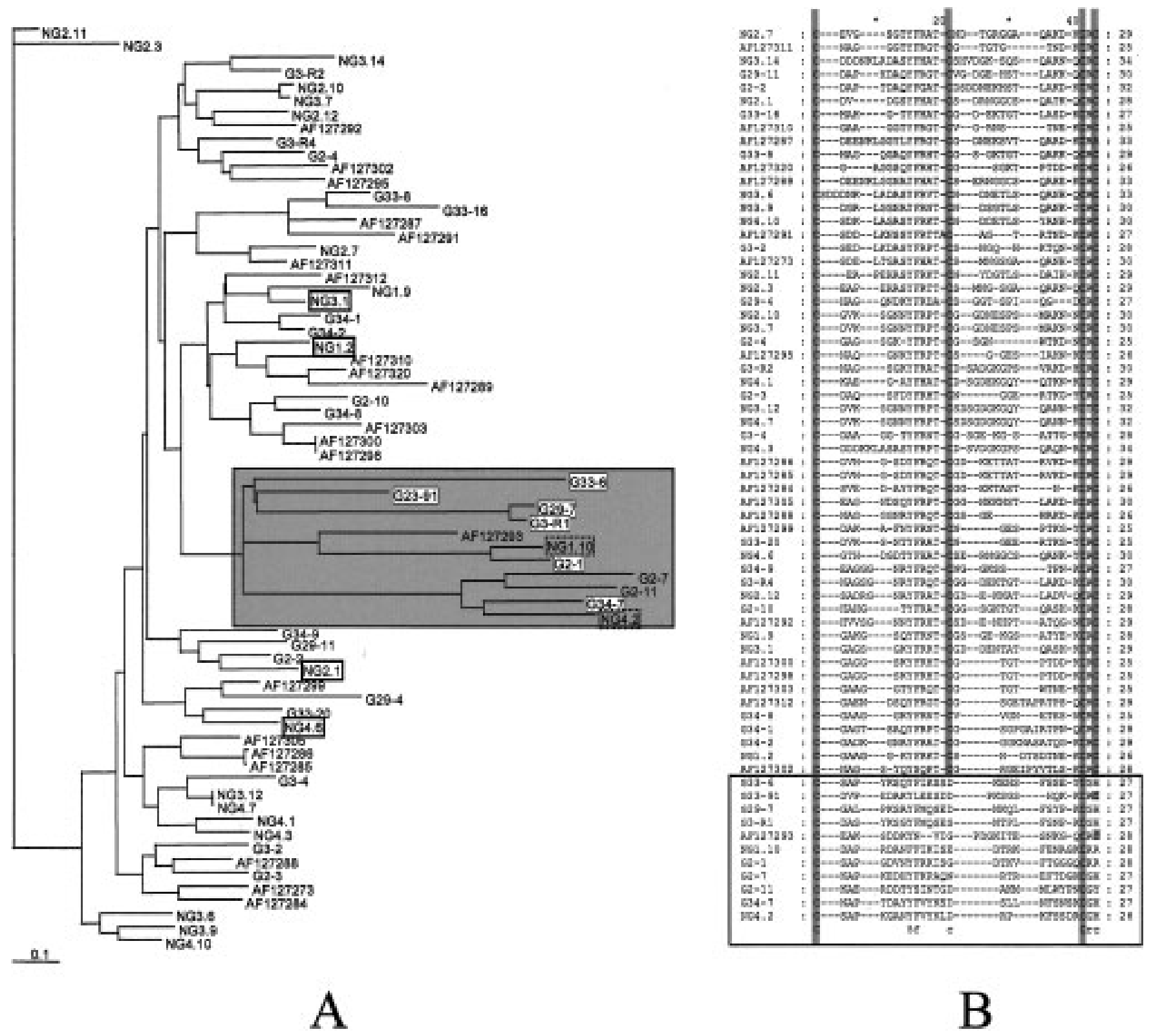

Fig. 1. DBL-1 $\alpha$ var sequences expressed in patients with severe malaria revealed predominantly expressed sequences lacking 1-2 cysteine residues. (A) Maximum likelihood phylogenetic tree of var genes based on the N-terminal segment of DBLl $\alpha$. The value of the gamma distribution with shape parameter $\alpha$ was 1.6315. Sequences preferentially (closed boxes) and rarely (open boxes) expressed in each isolate are indicated. Abbreviations: AF, accession numbers of GenBank from Sudanese sequences; G, sequences obtained from parasites of severe malaria; NG, sequences obtained from parasites of mild malaria. The gray area represents the group of sequences lacking 1-2 cysteine residues. (B) Alignment of 67 deduced amino acid sequences from the cysteine-rich region of DBLl $\alpha$ var sequences from Brazilian patients with severe malaria (G), mild malaria (NG), and patients from Sudan (AF). The region of DBL $1 \alpha$ domain lacking 1-2 cysteine residues from 11 sequences is boxed.

the PfEMPl DBLl $\alpha$ domain (12-15). We thus investigated whether a DBLl $\alpha$ domain from the most abundantly expressed sequence of a patient with severe malaria could bind to erythrocytes. To do so, the DBL $1 \alpha \mathrm{N}$-terminal domain from the P. falciparum isolate G2 (clone 1) (AF368922) was expressed with a $6 \times$-histidine tail in E. coli and used in IFA analysis. A similar methodology had been previously used to demonstrate PfEMPI as the rosetting ligand of $P$. falciparum (13). As positive and negative controls, a polyclonal mouse anti-human erythrocyte antibody and a nonrelated His-tagged protein from Leishmania were used, respectively. Similar to the positive control, the DBLl $\alpha$-His gave a distinct surface staining with all uninfected erythrocytes (Fig. 3A and 3B). In contrast, no staining was 
A
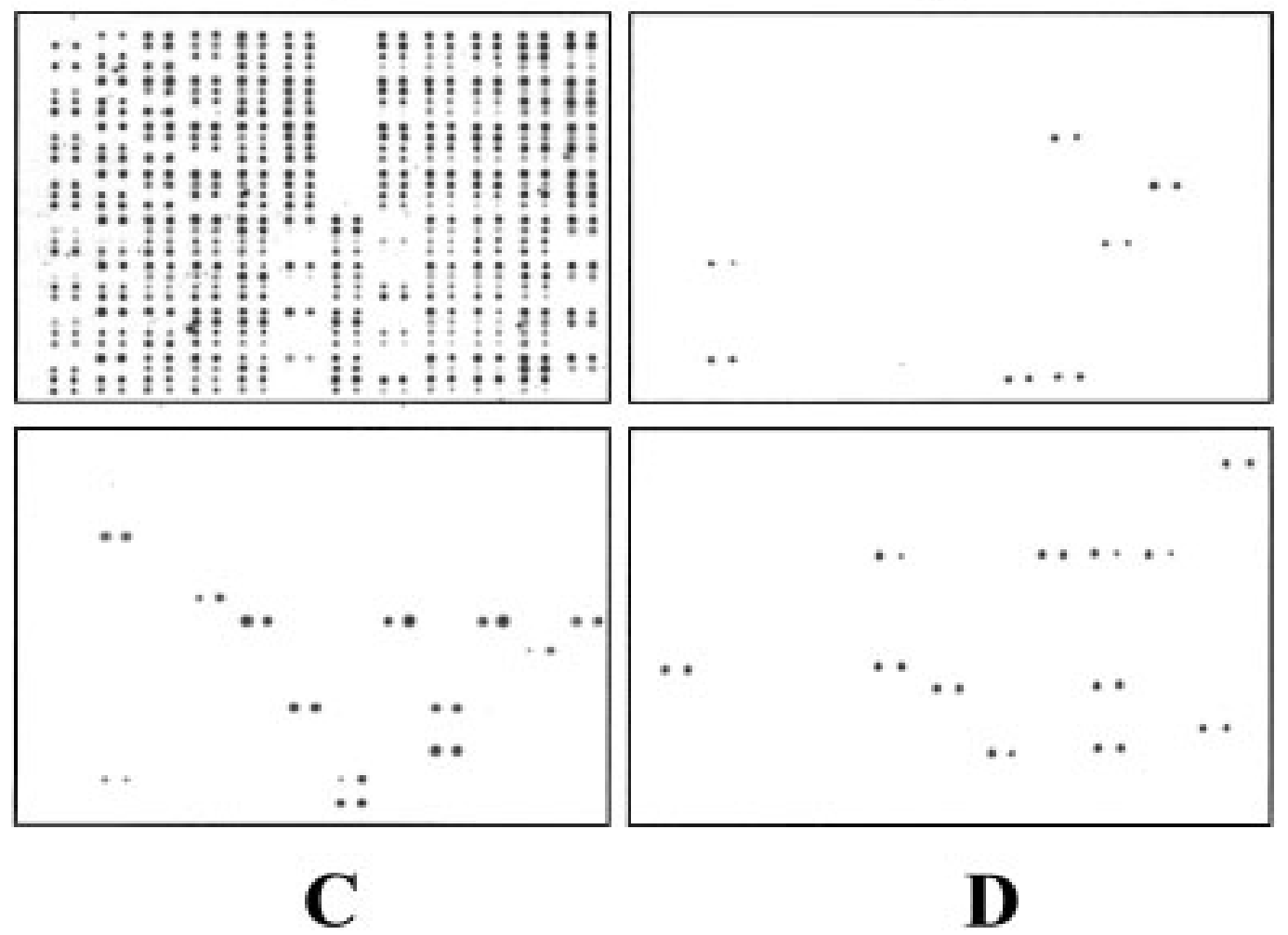

Fig. 2. DBLl $\alpha$ var sequences expressed in severe malaria parasites are frequently present in the var gene repertoire of mild malaria parasites. High-density filters containing $320 \mathrm{DBL} 1 \alpha$ var sequences obtained from parasites of patients with mild malaria were spotted in duplicates and hybridized with a plasmid-specific probe, T7 (A) or probes obtained from severe malaria parasites: (B) G29-7, (C) G33-6, or (D) G34-7. All spaces with no signal hybridization in A correspond to negative controls (water).

detected with an unrelated Leishmania Metal-His protein (Fig. 3C).

\section{Discussion}

An understanding of the molecular mechanisms underlying severe noncerebral falciparum malaria should lead to rational and novel control strategies. Towards this end, we analyzed expressed P. falciparum DBLl $\alpha$ var sequences from patients with severe noncerebral malaria from the Brazilian Amazon. Twentythree different and previously unreported deduced amino acid sequences were obtained from parasites obtained from these patients. Significantly, all the patients with severe malaria harbored predominantly expressed PfEMPI DBLl $\alpha$ sequences displaying a region lacking 1-2 cysteine residues that subgrouped them in clustal alignments. This subgroup was maintained when a similar analysis was performed using expressed sequences obtained from parasites of patients with mild malaria and sequences from this same var gene segment of isolates from Sudan available at GenBank. High-density filters representing genomic var DBLl $\alpha$ sequences of parasites obtained from 80 patients with mild malaria from four different regions in the Brazilian Amazon, revealed that
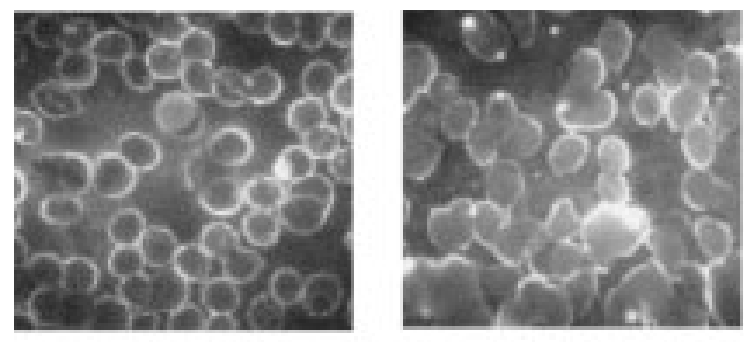

A
B

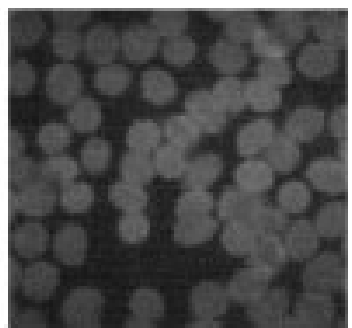

C
Fig. 3. A DBLl $\alpha$ domain expressed in bacteria binds directly to erythrocytes. IFA analysis of uninfected erythrocytes using a polyclonal mouse anti-human erythrocyte antibody (A), recombinant protein DBLl $\alpha$ (G2-1)-His (B) or a nonrelated His-tagged protein from Leishmania (C). 
these sequences are widely distributed; yet, they are predominantly expressed in patients with severe noncerebral malaria. This is the first report demonstrating an association between severe noncerebral malaria in patients from Brazil and particular PfEMP1 DBLl $\alpha$ sequences.

The results and conclusions drawn in this study were based on parasite material obtained from six patients diagnosed with severe noncerebral falciparum malaria. To identify these patients, we surveyed the clinical histories of 1599 patients with falciparum malaria kept at the Núcleo de Estudos em Malária da Superintendência de Controle de Endemias do Estado de São Paulo, Brasil (SUCEN) from 1987-2000. This laboratory is responsible for the diagnosis and treatment of all reported malaria cases in the city of São Paulo, a Brazilian non-endemic region. Most telling, it holds the highest number of clinical records of patients with severe malaria in Brazil. We were only able to identify six patients with the criteria determined by WHO to diagnose severe malaria. In addition to these criteria, we also demonstrated higher than normal PCT serum levels in four of these patients; this new parameter has been recently proposed to correlate disease severity and risk of mortality in malaria (22). These numbers thus accurately reflect the scarcity of patients with severe malaria in Brazil and reinforce the biological value of the data obtained in this study.

It is now well established that there is relaxed transcription of most if not all var genes during ring stages. Thus, even with highly purified samples containing mostly trophozoite and schizonts used in the RNA extractions, we cannot exclude the possibility of minor contaminations with ring stages. Moreover, malarial infections are not clonal, even in regions of low endemicity. Therefore, it is not surprising to identify different var DBLl $\alpha$ transcripts in parasites obtained from the patients that participated of this study. Remarkably, however, there were predominantly expressed PfEMPI DBLl $\alpha$ sequences in all parasites obtained from the six patients with severe malaria. Moreover, these sequences displayed a common region lacking 1-2 cysteine residues toward their C-termini that allowed them to be subgrouped in dendogram analysis.

To validate this result, we performed a similar analysis including expressed DBLl $\alpha$ sequences of parasites from patients with mild malaria and DBLl $\alpha$ sequences from this same gene segment from isolates of Sudan available at GenBank. Although the sequences of two clones from two patients with mild malaria lacked these cysteine residues, they represented only 1 out of 14 clones sequenced from each patient. Similarly, one sequence from Sudan also subgrouped in this analysis; unfortunately, we have no idea of the clinical status of this patient or whether this sequence is expressed. Worth of mentioning, because of their lower parasitemias, we cultured the parasites from the Brazilian patients with mild malaria for three cycles before extracting their RNA. var genes switch at a frequency of approximately $2 \%$ (34). Moreover, there is a preference to switch back to the previously expressed var gene (35). Thus, it is highly unlikely that after these three cycles a predominantly expressed DBLl $\alpha$ sequence could have been replaced. These data demonstrate that patients with mild malaria predominantly expressed DBLl $\alpha$ domains having all four cysteine residues in this region of the domain. In contrast, patients with severe noncerebral malaria harbor parasite populations predominantly expressing $\mathrm{DBLl} \alpha$ domains lacking 1-2 cysteine residues. In fact, patient G3 at the moment of having severe malaria had predominant DBLl $\alpha$ sequences lacking two cysteine residues; 20 days later at a recrudescence with mild malaria, the predominant DBLl $\alpha$ sequences displayed four cysteine residues. It is thus tempting to speculate that a different folding of these domains confer the parasite populations expressing them different degrees of virulence and reinforce the importance of PfEMPI domain folding in the pathology associated with malaria.

Two main phenomena have been associated with severe malaria: cytoadherence and rosetting. Of them, rosetting has been unequivocally demonstrated to be associated with particular sequences of the PfEMPI DBLl $\alpha$ domain (12-15). Here, we demonstrate that a DBLl $\alpha$ domain representing the most abundantly expressed PfEMPI DBLl $\alpha$ sequence from one of the patients with severe falciparum malaria expressed in bacteria binds to erythrocytes. Similar results have been reported using a GST-tagged var DBLl $\alpha$ domain from an isolate displaying all four cysteine residues (13). These results strongly suggest that this domain could induce rosetting in Brazilian field isolates; unfortunately, there are no reports of this phenomenon from Brazil, but clearly these studies are required to determine the role of rosetting in falciparum infections from this geographical region.

A recent study demonstrated the association of severe malaria with a particular var DBL $\delta$ sequences in patients from French Guyana (20). Together with the studies reported here, these data indicate that there are indeed associations of severe malaria with particular PfEMPI sequences. However, in addition to the var multigene family, many other multigene families such as rif, stevor, clag, and pf60, likely involved in virulence, have been discovered in P. falciparum (reviewed in 36). High throughput methodologies can now be envisioned to discover and compare the repertoire of genomic and expressed virulent genes circulating in endemic regions with particular clinical syndromes of severe malaria. These data in turn should help in elucidating the molecular mechanisms underlying the different clinical manifestations to develop rationale alternative eradication control strategies. 


\section{Acknowledgments}

We thank Dr. Alex Fiorini Carvalho and Dr. Luiz Fernando Lima Reis (Institute Ludwig) for facilitating the use of robotics to spot the filters. We thank Drs. Marcelo Urbano Ferreira and Crispim Cerutti Jr. for some samples from patients with mild malaria; Dr. Emilia Kimura for the anti-human erythrocyte polyclonal mouse antibody; and Dr. Silvia Reni Uliana for the Metal-His protein. This work received support from the INCO-DC Programme (IC18-CT98-0364).

\section{References}

1. Miller LH, Good MF, Milon G. (1994) Malaria pathogenesis. Science 264: 1878-1883.

2. Su XZ, Heatwole VM, Wertheimer SP, et al. (1995) The large diverse gene family var encodes proteins involved in cytoadherence and antigenic variation of Plasmodium falciparuminfected erythrocytes. Cell 82: 89-100.

3. Cooke B, Coppel R, Wahlgren M. (2000) Falciparum malaria: sticking up, standing out and out-standing. Parasitol. Today 16: 416-420.

4. Handunnetti SM, David PH, Perera KL, Mendis KN. (1989) Uninfected erythrocytes form "rosettes" around Plasmodium falciparum infected erythrocytes. Am. J. Trop. Med. Hyg. 40: 115-118.

5. MacPherson GG, Warrell MJ, White NJ, Looareesuwan S, Warrell DA. (1985) Human cerebral malaria. A quantitative ultrastructural analysis of parasitized erythrocyte sequestration. Am. J. Pathol. 119: 385-401.

6. Aikawa M. (1988) Human cerebral malaria. Am. J. Trop. Med. Hyg. 39: 3-10.

7. Carlson J, Helmby H, Hill AV, Brewster D, Greenwood BM, Wahlgren M. (1990) Human cerebral malaria: association with erythrocyte rosetting and lack of anti-rosetting antibodies. Lancet 336: 1457-1460.

8. Pongponratn E, Riganti M, Punpoowong B, Aikawa M. (1991) Microvascular sequestration of parasitized erythrocytes in human falciparum malaria: a pathological study. Am. J. Trop. Med. Hyg. 44: 168-175.

9. Treutiger CJ, Hedlund I, Helmby H, et al. (1992) Rosette formation in Plasmodium falciparum isolates and anti-rosette activity of sera from Gambians with cerebral or uncomplicated malaria. Am. J. Trop. Med. Hyg. 46: 503-510.

10. Rowe A, Obeiro J, Newbold CI, Marsh K. (1995) Plasmodium falciparum rosetting is associated with malaria severity in Kenya. Infect. Immun. 63: 2323-2326.

11. Newbold C, Warn P, Black G, et al. (1997) Receptor-specific adhesion and clinical disease in Plasmodium falciparum. Am. J. Trop. Med. Hyg. 57: 389-398.

12. Rowe JA, Moulds JM, Newbold CI, Miller LH. (1997) P. falciparum rosetting mediated by a parasite-variant erythrocyte membrane protein and complement-receptor 1. Nature 388: 292-295.

13. Chen Q, Barragan A, Fernandez V, et al. (1998) Identification of Plasmodium falciparum erythrocyte membrane protein 1 (PfEMP1) as the rosetting ligand of the malaria parasite P. falciparum. J. Exp. Med. 187: 15-23.

14. Chen Q, Heddini A, Barragan A, Fernandez V, Pearce SF, Wahlgren M. (2000) The semiconserved head structure of Plasmodium falciparum erythrocyte membrane protein 1 mediates binding to multiple independent host receptors. J. Exp. Med. 192: $1-10$.

15. Barragan A, Fernandez V, Chen Q, von Euler A, Wahlgren M, Spillmann D. (2000) The duffy-binding-like domain 1 of
Plasmodium falciparum erythrocyte membrane protein 1 (PfEMPI) is a heparan sulfate ligand that requires 12 mers for binding. Blood 95: 3594-3599.

16. WHO. (2000) Severe falciparum malaria. Trans. R. Soc. Trop. Med. Hyg. 94(suppl 1): S1-S90.

17. Duarte MI, Corbett CE, Boulos M, Amato Neto V. (1985) Ultrastructure of the lung in falciparum malaria. Am. J. Trop. Med. Hyg. 34: 31-35.

18. Corbett CE, Duarte MI, Lancellotti CL, Silva MA, Andrade Jr HF. (1989) Cytoadherence in human falciparum malaria as a cause of respiratory distress. J. Trop. Med. Hyg. 92: 112-120.

19. Boulos M. (1992) Clinical picture of severe malaria. Rev. Inst. Med. Trop. Sao Paulo 34(Suppl 9): S41-S42.

20. Ariey F, Hommel D, Le Scanf C, et al. (2001) Association of severe malaria with a specific Plasmodium falciparum genotype in French Guiana. J. Infect. Dis. 184: 237-241.

21. Kirchgatter K, Mosbach R, del Portillo HA. (2000) Plasmodium falciparum: DBL-1 var sequence analysis in field isolates from central Brazil. Exp. Parasitol. 95: 154-157.

22. Chiwakata CB, Manegold C, Bonicke L, Waase I, Julch C, Dietrich M. (2001) Procalcitonin as a parameter of disease severity and risk of mortality in patients with Plasmodium falciparum malaria. J. Infect. Dis. 183: 1161-1164.

23. Trager W, Jensen JB. (1976) Human malaria parasites in continuous culture. Science 193: 673-675.

24. Pasvol G, Wilson RJ, Smalley ME, Brown J. (1978) Separation of viable schizont-infected red cells of Plasmodium falciparum from human blood. Ann. Trop. Med. Parasitol. 72: 87-88.

25. Ferreira MU, Liu Q, Kaneko O, et al. (1998) Allelic diversity at the merozoite surface protein-1 locus of Plasmodium falciparum in clinical isolates from the southwestern Brazilian Amazon. Am. J. Trop. Med. Hyg. 59: 474-480.

26. Kyes S, Pinches R, Newbold C. (2000) A simple RNA analysis method shows var and rif multigene family expression patterns in Plasmodium falciparum. Mol. Biochem. Parasitol. 105: 311-315.

27. Taylor HM, Kyes SA, Harris D, Kriek N, Newbold CI. (2000) A study of var gene transcription in vitro using universal var gene primers. Mol. Biochem. Parasitol. 105: 13-23.

28. Boyle JS, Lew AM. (1995) An inexpensive alternative to glassmilk for DNA purification. Trends Genet. 11: 8.

29. Altschul SF, Madden TL, Schaffer AA, et al. (1997) Gapped BLAST and PSI-BLAST: a new generation of protein database search programs. Nucleic Acids Res. 25: 3389-3402.

30. Thompson JD, Gibson TJ, Plewniak F, Jeanmougin F, Higgins DG. (1997) The CLUSTAL_X windows interface: flexible strategies for multiple sequence alignment aided by quality analysis tools. Nucleic Acids Res. 25: 4876-4882.

31. Posada D, Crandall KA. (1998) MODELTEST: testing the model of DNA substitution. Bioinformatics 14: 817-818.

32. Strimmer K, von Haeseler A. (1996) Quartet puzzling: a quartet maximum-likelihood method for reconstructing tree topologies. Mol. Biol. Evol. 13: 964-969.

33. Swofford DL. (1998) PAUP*. Phylogenetic Analysis Using Parsimony (*and Other Methods). Version 4, Sinauer Associates Sunderland, MA.

34. Roberts DJ, Craig AG, Berendt AR, Pinches R, Nash G, Marsh K, Newbold CI. (1992) Rapid switching to multiple antigenic and adhesive phenotypes in malaria. Nature 357: 689-692.

35. Chen Q, Fernandez V, Sundstrom A, Schlichtherle M, Datta S, Hagblom P, Wahlgren M. (1998) Developmental selection of var gene expression in Plasmodium falciparum. Nature 394: 392-395.

36. Kyes S, Horrocks P, Newbold C. (2001) Antigenic variation at the infected red cell surface in malaria. Annu. Rev. Microbiol. 55: 673-707. 